\title{
Automatic Identification of a Particular Vertebra in the Spinal Column Using Surface-Based Registration
}

\author{
Jeannette L. Herring and Benoît M. Dawant \\ Department of Electrical and Computer Engineering, \\ Vanderbilt University, Nashville, TN 37235 \\ jnet@vuse.vanderbilt.edu
}

\begin{abstract}
In this work, we register vertebral points collected in physical space to spinal column surfaces extracted from computed tomography images. The vertebral points are taken from a single vertebra to represent the region of surgical interest. The surface is automatically extracted using an iso-intensitybased algorithm and thus contains multiple vertebrae. This work introduces an enhancement to currently existing methods of intra-operative vertebral registration by allowing the portion of the spinal column surface that correctly matches a set of physical vertebral points to be automatically selected from several possible choices. We find the correct portion of the surface by registering the set of physical points to multiple surface areas, including all vertebral surfaces that potentially match the physical point set. We then compute the standard deviation of the surface error for the set of points registered to each vertebral surface that is a possible match, and the registration that corresponds to the lowest standard deviation designates the correct match. We have performed our current experiments on two plastic spine phantoms.
\end{abstract}

\section{Introduction}

Health problems associated with the lumbar region of the spine are prevalent, potentially debilitating, and often quite expensive to treat. In fact, more than 250,000 lumbar spine operations are performed annually in the United States at a cost of more than $\$ 6$ billion per year [6]. Because of the prevalence of lumbar back problems, any improvement in surgical procedures can have a tremendous clinical impact. We are particularly interested in contributions provided by image-guided surgical techniques.

Improving the feasibility of image-guided back surgery by performing a real-time registration of vertebrae in different image modalities or in image and physical space is an active research area. Clinical studies have been done to show that the technique is a valid approach for the insertion of pedicle screws (for example, see [2] and [3]). Image-guided surgical techniques have also been used successfully to perform lumbar discectomies (for example, see [14]) and spinal biopsies (for example, see [16]).

In addition to the clinical studies that have been done in the area of image-guided vertebral surgery, several technical studies have been performed to investigate various methods of vertebral segmentation and registration. In particular, Lavallée and colleagues have done a substantial amount of work in this area. Their studies show that sub-millimetric registration accuracy can be achieved between points obtained 
intra-operatively (using an optically tracked ultrasound probe, a 3-D pointer, and projection X-ray images [8]) and surfaces extracted pre-operatively from CT images (using modified snake and spline techniques [10]). Points are registered to surfaces by minimizing the energy required to make projection lines from 2-D contours tangent to the 3-D surface when using $\mathrm{x}$-ray images and by applying more traditional surface-based techniques when using 3-D methods of point collection [7,8]. Results reported so far using automatic or semi-automatic surface generation techniques have been achieved primarily on single isolated vertebrae [9].

An important premise of the clinical and technical studies is that the physical points must be collected from the correct vertebra; that is, from the vertebra that is involved in the surgical procedure or the registration trial. In clinical situations, this intra-operative identification of the correct vertebra can be a challenging task, especially for closed-back, minimally invasive procedures. It requires the surgeon to count vertebrae by feeling spinous processes through the skin, a procedure that is complicated by the presence of fatty tissues. Because this manual identification process introduces the possibility of error, it would be helpful to have a registration algorithm that could automatically locate the vertebra of interest.

Our research demonstrates that surface-based registration methods can be used to identify which vertebral surface in a CT image volume containing multiple vertebrae correctly matches a physical point set representing a single vertebra. To perform the surface-based registration, we use a method based upon the iterative closest point algorithm developed by Besl and McKay [1]. To segment the vertebral surface, we use the fully automatic marching cubes algorithm proposed by Lorensen and Cline [11]. Since each vertebra has a slightly different shape, measurements related to the quality of the registration results indicate which vertebral surface in the image volume best corresponds to the physical point set.

\section{Methods}

\subsection{Image Acquisition}

We have performed our experiments on two plastic phantoms of the spine. The first phantom, which we have designated Phantom I, is a life-size model of the entire spinal column. The second phantom, designated Phantom II, is a life-size model of the lumbar portion of the spinal column. Both phantoms are manufactured by the Carolina Biological Supply Company in Burlington, North Carolina.

CT scans of the two phantoms were obtained using a Philips Tomoscan AV scanner. For both phantoms, the image volume contains 70 slices with a slice thickness of $2 \mathrm{~mm}$. Every slice has 512 x 512 voxels with voxel dimensions in millimeters of $0.625 \times 0.625 \times 2$. Both CT image volumes used in this study were acquired as stacks of image slices with no inter-slice gap or slice overlap. The gantry tilt angle during image acquisition was zero. Phantom I was placed in the scanner to ensure that complete images of L1 and L2 were obtained, and Phantom II was placed in the scanner to ensure that complete images of L3, L4, and L5 were obtained. 


\subsection{Triangulated Surface Extraction}

A triangle set representation of the surface of the spinal column was automatically extracted from each CT scan using an independently implemented version of the classic marching cubes method developed by Lorensen and Cline [11]. The number of triangles in the resulting mesh was reduced using the decimation algorithm from the commercially available Visualization Toolkit (VTK) [15].

The only parameter required by the marching cubes algorithm is an intensity value. This algorithm is well suited to the extraction of bone surfaces in CT images, since the intensity level of bone is generally an order of magnitude greater than the intensity levels of all other materials. Of course, the method results in the inclusion of overlapping parts of neighboring vertebrae, but the extraneous surface information does not appear to be a problem for the surface-based registration process we use.

Hounsfield numbers of the plastic phantoms range from approximately 600 to approximately 800, with Phantom II having a slightly higher Hounsfield number than Phantom I. The Hounsfield number of the air surrounding the phantom is -1024 . There is an offset of 1024 introduced into the intensity values due to the method used to transfer the scans from the CT machine, resulting in average intensity values of about 1600 to 1800 for the plastic and 0 for the surrounding air. Thus, iso-surfaces were created for the phantom scans at the intermediate intensity values of 800 for Phantom I and 900 for Phantom II.

\subsection{Surface Point Identification}

The physical space coordinates of approximately 300 surface points were acquired for each vertebra using a three-dimensional spatial localizer (3DSL) consisting of a probe and an Optotrak 3020 system (Northern Digital, Ontario, Canada). These points were collected by sweeping the 3DSL over the vertebral surface, a method which allows hundreds of points to be acquired in a few seconds. The point collection process was performed by an engineering graduate student with advice from a general surgeon.

For each vertebra, the collected surface points represent the central laminar regions and the tip of the spinous process. In previous work, we have studied the sensitivity of surface-based registration to different regions of the posterior vertebral surface, and we have shown that points from the central laminar regions and the tip of the spinous process provide sufficient information for accurate surface-based registration [4].

\subsection{Registration}

We perform surface-based registration of physical points from each lumbar vertebra to the spinal column surfaces extracted from the CT scans of Phantom I and Phantom II. That is, we attempt to register physical points collected from vertebra L1 to surfaces corresponding to all five lumbar vertebrae, and we repeat the process for L2 through L5. Both spinal column surfaces contain multiple vertebrae, so our registration trials address the question of whether the vertebral surface that correctly matches our physical point set can be selected out of several choices.

To perform our registration trials, we use an independent implementation [12] of the iterative closest point algorithm of Besl and McKay [1]. The method is a two-step 
process. First, the closest point on one surface is computed for each point in a set of points representing the other surface. (In this study, the first surface is a triangle set representation of the surface in the $\mathrm{CT}$ image, and the point set representation of the second surface is a set of physical-space surface points.) Second, a transformation is determined by registering these two point sets. This process is iterated until some stopping criterion is satisfied. The method converges to a local minimum of the cost function, which is the root-mean-square (RMS) distance between the corresponding points at the last iteration. Because the physical-space surface points we record are the positions of the center of the ballpoint tip of the 3DSL, the recorded surface points are displaced from the actual surface by the radius of the tip. We use the method described by Maurer and colleagues to correct for this displacement [13].

Because of the possibility of convergence to a local minimum that is not the correct solution, the algorithm works best when it is initialized with rotations and translations that are close to the exact solution. In this work, the initial registration for correctly matched vertebral points and surfaces is computed by aligning the principal axes of the physical surface points and the CT image surface. This registration is translated along the spinal column to compute the initial position of the point set in relation to other (mis-matched) vertebral surfaces. We use a translation of plus or minus $30 \mathrm{~mm}$ per vertebra through which the correct position is displaced. This method of finding different initial positions along the spinal column should translate directly into clinical work: The principal axes transformation can be used to find the initial position of the chosen vertebra, and that position can then be translated up and down the spinal column to test whether the chosen vertebra is actually the correct one.

\subsection{Error Computation}

Our method of assessing registration error requires the comparison of registration results with a gold standard [4]. Since the work reported herein investigates mismatched vertebral registrations, there is no associated gold standard. Thus, we report results in terms of surface error, which is computed as the RMS distance of the registered point set from the surface. It is important to distinguish surface error from registration error, since we have found that surface error is generally not a good predictor of registration error. However, our experiments show that surface error along with its standard deviation can provide a relative measure of how well the shape of the point set matches the shape of the surface. Our primary concern for this particular set of experiments is finding the closest match between the two shapes, so surface error and its standard deviation are appropriate error computation methods.

\section{Results}

Figure 1 shows the surface extracted from Phantom I. From top to bottom, the figure shows part of a thoracic vertebra, a complete thoracic vertebra, vertebrae L1 and L2 (which can be identified by the fiducial markers in the right central laminar regions), L3, and part of L4. Note that the point sets we have chosen to use in our registration trials - the central laminar regions and the spinous process tips - have rather similar shapes, especially for neighboring vertebrae. The greatest visual 
variation tends to be in the transverse processes, which are not included in our physical point set. Also, note that the markers visible in Figure 1 are not used in this particular experiment; they are related to other registration trials we have conducted. A similar surface containing vertebrae L2 through L5 and part of the sacrum exists for Phantom II.

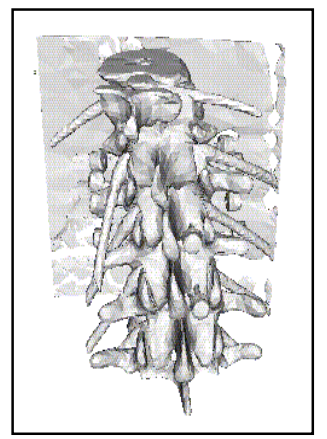

Fig. 1. Surface of spinal column extracted from CT scan of Phantom I. The surface contains parts of two thoracic vertebrae and four lumbar vertebrae.

Figure 2 shows the RMS surface errors obtained in our registration trials. Each bar represents the result of registering the physical point set designated by the legend to the vertebral surface shown along the $\mathrm{x}$ axis. Recall from the Methods section that the surface points are displaced from the actual surface by the radius of the ballpoint tip of the 3DSL, which is $0.5 \mathrm{~mm}$. Our program to compute surface distance does not correct for this displacement, so the RMS surface error should be $0.5 \mathrm{~mm}$ in Figure 2 for a "perfect" fit.

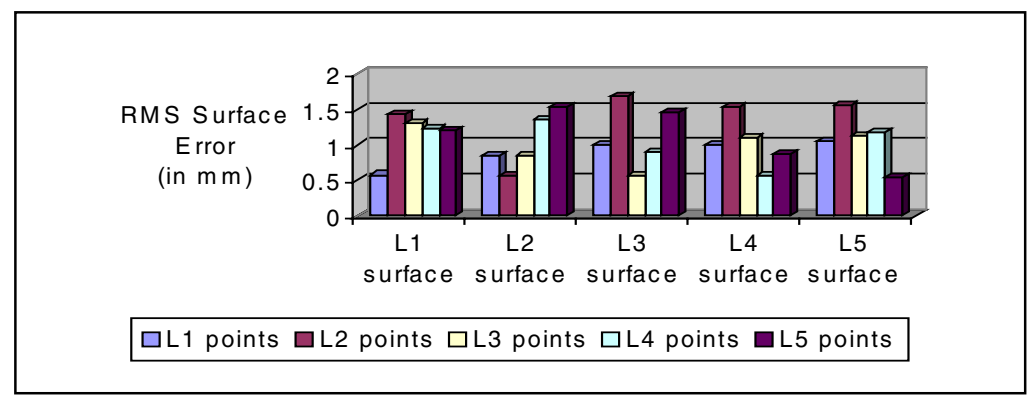

Fig. 2. RMS surface errors for physical point sets shown in the legend registered to vertebral surfaces shown along the $\mathrm{x}$ axis. All errors are in millimeters.

Without exception, Figure 2 shows that the correctly matched points and surfaces yield the lowest RMS surface errors. However, it is interesting to note that the RMS surface errors for mis-matched vertebrae are also consistently low, even when the two vertebrae being matched have quite different shapes, as is the case for L1 and L5 in this particular experiment. In fact, certain incorrect matches provide surface errors that are not dramatically higher than the errors generated by correct matches. For 
example, consider the results of registering points from L1, L2, and L3 to the surface of L2. The L2-to-L2 match has the lowest RMS surface error at $0.56 \mathrm{~mm}$, but the L1to-L2 and L3-to-L2 mis-matches both have surface errors of $0.85 \mathrm{~mm}$, which is also a relatively low value.

The fact that RMS surface errors are relatively low for mis-matched points and surfaces suggests that this error may not be the best indicator of a vertebral mismatch. This observation led us to examine the standard deviation of surface error as a possible indicator. Figure 3 shows the standard deviation for each registration trial. As in Figure 2, each bar represents the registration result obtained for the physical point set designated by the legend and the vertebral surface shown along the $\mathrm{x}$ axis. As before, the correctly matched points and surfaces yield the lowest standard deviations in all cases. However, the spread is greater in this chart, suggesting that the standard deviation is a better predictor of a correct match than the RMS surface error.

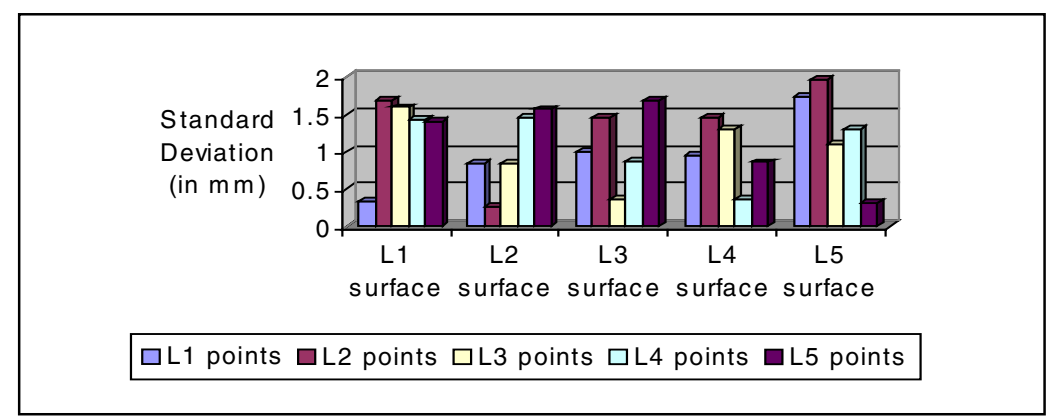

Fig. 3. Standard deviations of surface errors for physical point sets shown in the legend registered to vertebral surfaces shown along the $\mathrm{x}$ axis. All errors are in millimeters.

\section{Discussion}

It is not surprising that the correct match yields the lowest RMS surface error in Figure 2, but it is rather unexpected to find such small surface errors for mis-matched vertebrae. This finding is due to fact that the registration algorithm is designed to minimize distance between the point set and the surface, the very distance which also serves as our definition of surface error. In other words, because the behavior of the registration algorithm is controlled by point-to-surface distance, it will attempt to place the point set close to some surface (resulting in a relatively low RMS surface error), but there is no guarantee that the point set will be close to the correct surface.

As Figure 3 shows, a better predictor of a correct match is the standard deviation of surface error. Even though the registration algorithm attempts to place the entire set of registered points close to some surface, a mis-match between the point set shape and the surface shape results in less consistency among individual point-to-surface distances, or in other words, a larger standard deviation. For a correctly registered case, the excellent match between the shape of the point set and the shape of the 
surface results in a greater consistency among distances from the points to the surface, or in other words, a smaller standard deviation.

Whether or not the results we have obtained on phantom data can be verified on patient data remains to be tested. However, we have performed preliminary studies of the effectiveness of using an iso-intensity-based triangulation algorithm to segment vertebrae in patient data sets, and we have achieved satisfactory results [5]. We believe that shape differences among vertebrae in patient data sets will be at least as pronounced as differences among the phantom vertebrae we have used. Thus, it is likely that the observations we have made on these phantoms will apply to real data sets. We also note that there are other measures of comparison between point set and surface shapes that can be used if the standard deviation of the surface error proves to be insufficient. For example, additional experiments not included in this paper show that the use of physical points collected from the transverse processes and not used in registration can provide a clear distinction between matched and mis-matched vertebrae. In our trials using these supplementary points, correct matches have submillimetric RMS surface errors and incorrect matches have RMS surface errors of 4 to $6 \mathrm{~mm}$.

It should be noted that knowledge of whether a correct match exists is required prior to surgical exposure of the physical points. For this reason, we are investigating the possibility of collecting the point set percutaneously from ultrasound images. Preliminary experiments suggest that registration results achieved using ultrasound point sets will be similar to those achieved using physical point sets.

\section{Conclusion}

Our experiments show that the standard deviation of surface error can be used to select the correct match between a set of vertebral surface points collected in physical space and a surface of the spinal column extracted from a CT scan of multiple vertebrae. The ability to make this determination automatically removes the need for manual identification of matching vertebrae in image and physical space. In the future, we will run similar experiments with patient scans and points collected via ultrasound. We will also test our findings on CT scans with larger slice spacings.

\section{Acknowledgements}

This work has been supported in part by an NDSEG fellowship awarded through the Office of Naval Research and by NIH grant 5R01-G752798-03. The authors gratefully acknowledge the many contributions of Alan Herline, M.D., who provided invaluable input from a surgeon's point of view, and Calvin Maurer, who provided the executable code for the registration algorithm. They would also like to express their appreciation to Diane Muratore, who helped collect the physical point sets, and Allen Jackson, who provided the CT scans used in our study. 


\section{References}

1. Besl, P.J. and McKay, N.D., "A Method for Registration of 3-D Shapes." IEEE Transactions on Pattern Analysis and Machine Intelligence 14, pp. 239-256, 1992.

2. Carl, A.L., Khanuja, H.S., Sachs, B.L., Gatto, C.A., vomLehn, J., Vosburgh, K., Schenck, J., Lorensen, W., Rohling, K., and Disler, D., "In Vitro Simulation: Early Results of Stereotaxy for Pedicle Screw Placement." Spine 22, pp. 1160-1164, 1997.

3. Foley, K.T. and Smith, M.M., "Image-guided Spine Surgery." Neurosurgery Clinics of North America 7, pp. 171-186, 1996.

4. Herring, J.L., Dawant, B.M., Maurer, C.R., Jr., Muratore, D.M., Galloway, R.L., Jr., and Fitzpatrick, J.M., "Surface-based Registration of CT Images to Physical Space for Imageguided Surgery of the Spine: A Sensitivity Study." IEEE Transactions on Medical Imaging 17, pp. 743-752, 1998.

5. Herring, J.L., Maurer, C.R., Jr., and Dawant, B.M., "Sensitivity Analysis for Registration of Vertebrae in Ultrasound and Computed Tomography Images." Proceedings of the SPIE Conference on Image Processing (San Diego, California) 3338, pp. 95-106, 1998.

6. Krause, T.M., "Case Management through a Multidisciplinary Spinal Evaluation." Orthopaedic Nursing 16, pp. 46-50, 1997.

7. Lavallée, S. and Szeliski, R., "Recovering the Position and Orientation of Free-Form Objects from Image Contours Using 3D Distance Maps." IEEE Transactions on Pattern Analysis and Machine Intelligence 17, pp. 378-390, 1995.

8. Lavallée, S., Szeliski, R., and Brunie, L. "Anatomy-Based Registration of ThreeDimensional Medical Images, Range image, X-Ray Projections, and Three-Dimensional Models Using Octree-Splines." In: Computer-integrated Surgery: technology and clinical applications, eds. R. Taylor, S. Lavallée, G. Burdea, and R. Mösges. Boston, Massachusetts: Academic Press, 1996. pp. 115-143.

9. Lavallée, S., Troccaz, J., Sautot, P., Mazier, B., Cinquin, P., Merloz, P., and Chirossel, J.P. "Computer-Assisted Spinal Surgery Using Anatomy-Based Registration." In: Computer-integrated Surgery: technology and clinical applications, eds. R. Taylor, S. Lavallée, G. Burdea, and R. Mösges. Boston, Massachusetts: Academic Press, 1996. pp. 425-449.

10. Leitner, F., Marque, I., Lavallée, S., and Cinquin, P. "Dynamic Segmentation: Finding the Edge With Snake Splines." In: Curves and Surfaces, eds. P.J. Laurent, A. Le Méhauté, and L.L. Schumaker. Boston, Massachusetts: Academic Press, 1991. pp. 279284.

11. Lorensen, W.E. and Cline, H.E., "Marching Cubes: A High Resolution 3D Surface Construction Algorithm." Computer Graphics (Anaheim, California) 21 [4], pp. 163-169, 1987.

12. Maurer, C.R., Jr., Aboutanos, G.B., Dawant, B.M., Maciunas, R.J., and Fitzpatrick, J.M., "A Method for Registration of 3-D Images Using Multiple Geometric Features." IEEE Transactions on Medical Imaging 15, pp. 836-849, 1996.

13. Maurer, C.R., Jr., Maciunas, R.J., and Fitzpatrick, J.M., "Registration of Head CT Images to Physical Space Using a Weighted Combination of Points and Surfaces." IEEE Transactions on Medical Imaging 17, pp. 753-761, 1998.

14. Mayer, H.M. and Brock, M., "Percutaneous endoscopic discectomy: surgical technique and preliminary results compared to microsurgical discectomy." Journal of Neurosurgery 78, pp. 216-225, 1993.

15. Schroeder, W., Martin, K., and Lorensen, B. The Visualization Toolkit, Upper Saddle River, New Jersey: Prentice Hall PTR, 1998.

16. Stringham, D.R., Hadjipavlou, A., Dzioba, R.B., and Lander, P., "Percutaneous Transpedicular Biopsy of the Spine." Spine 19, pp. 1985-1991, 1994. 